\title{
Electrophysiology of the Normal and Pathologic Muscle Activation
}

\author{
Guido Li Volsi* and Flora Licata
}

Department of Physiological Sciences, University of Catania, Italy

\begin{abstract}
Motorneurons are Central Processing Units able to generate sequences of action potentials whose frequency and duration are the result of algebraic sum of inward and outward currents crossing their membranes. On a macroscopic scale the activity of these Units will result in muscle contraction.

This lecture starts by focusing on the action potential, the motor unit and some integrative aspects of motor orders directed to spinal level. In fact, muscle contraction is subjected to multiple controls which take place at peripheral (proprioceptors), spinal (local circuits) and supraspinal (higher motor centers) levels. The excitation-contraction coupling, consisting in the switching-on of the neuromuscular synapsis, triggers the consequent mechanical event into the sarcomere. Exaggerated inward current in the motorneuron will produce hyperexcitability with consequent muscular rigidity (spasticity). Examples of incoming pathologic inputs to the motorneuron are analyzed and discussed.
\end{abstract}

Keywords: Muscle, electrophysiology, motor units, spasticity, action potential.

\section{PHYSIOLOGICAL ACTIVATION OF THE MOTOR UNIT}

The motor activity is the result of ordered sequences of muscle contractions that have an origin either voluntary or involuntary. Allen and Tsukahara in their notable paper "Cerebrocerebellar Communication System" [1] discussed several aspects related to the planning, initiation and execution of the movement. More specifically, a motor activity is controlled by two kinds of integrated processes. At spinal level the muscle contraction and the resulting movement are triggered by motorneuron activity which, in turn, depends on the reflex activity. Higher motor centers exert a finer and more tuned control on motorneurons by several neural networks including cortical premotor and motor areas, basal ganglia, thalamus, cerebellum and brain stem.

Therefore, under physiological conditions, the movement appears elegant, flowing and continuous, depending on a well-synchronized activation of the so-called motor units (MUs) ${ }^{1}$. In other words, a correct activation of a motorneuron, provided that membrane channels are "safe", will generate ionic currents able to produce trains of action potentials optimized in length and duration. These events will have an impact on neuromuscular junctions and, consequently, on sarcomeres.

Motorneurons and striated muscle fibers have common electrical features with some differences. Both generate action potentials whenever membrane depolarization reach the threshold following an inward $\mathrm{Na}^{+}$current. Nevertheless

*Address correspondence to this author at the Department of Physiological Sciences, University of Catania, Italy; Tel: +39 095738 4045; Fax: +39 095 738 4217; E-mails: glivolsi@unict.it, guidolivolsi@gmail.com

\footnotetext{
${ }^{1}$ The motor unit is the functional element of the Motor System. Charles Sherrington introduced the term motor unit to refer the motorneuron in the spinal cord and the population of muscle fibers that it innervates.
}

the duration of a single action potential in the motorneuron is less lasting (at lest 2-3 times) than that of the striated muscle fiber. Once the action potentials reach the terminals of the nervous fiber, they modulate voltage-dependent $\mathrm{Ca}^{2+}$ channels inducing an inward $\mathrm{Ca}^{2+}$ current which, in turn, leads to a release of acetylcholine. This "synaptic" acetylcholine binds and opens post-synaptic ionotropic channel-receptors (nicotinic channels) clustered at the endplate, and $\mathrm{Na}^{+} / \mathrm{K}^{+}$currents flow. Each receptor can bind two molecules of acetylcholine. The end-plate potential has a key significance for the correct excitability of the sarcolemma. In fact, the algebraic sum of the electrochemical forces for $\mathrm{Na}^{+}$and $\mathrm{K}^{+}$determine a local depolarization that triggers the action potential. The sarcomeres contract and generate mechanical events resulting in shortenings detectable (if discrete) on a macroscopic scale.

Muscle spindles and Golgi tendons are primarily involved in the control of the muscle contraction. In fact, muscle fibers must work at optimal length and avoid potentially damaging loads. The result will be an executed motor performance coherent with the planned one.

As above described, motorneurons are under the control of inputs coming from both the periphery and the higher motor centers (Fig. 1).

Interneurons are critical in influencing motorneuron activity. A malfunction of this circuit can produce heavy effects on muscle activity. In 1906 Sir Charles Sherrington published a collection of ten lectures that he had delivered at Yale University (USA) two years before. The title was: The Integrative Action of the Nervous System [2]. In the Lecture III Sherringhton focuses on the reciprocal inhibition, a new concept to describe a logic operation protocol in a joint. The excitation of an agonist muscle(s) is accompanied by a simultaneous inhibition of its antagonist(s). He was honored with the 1932 Nobel Price and Lord Adrian who shared the Price with him commented this new approach in the study of 


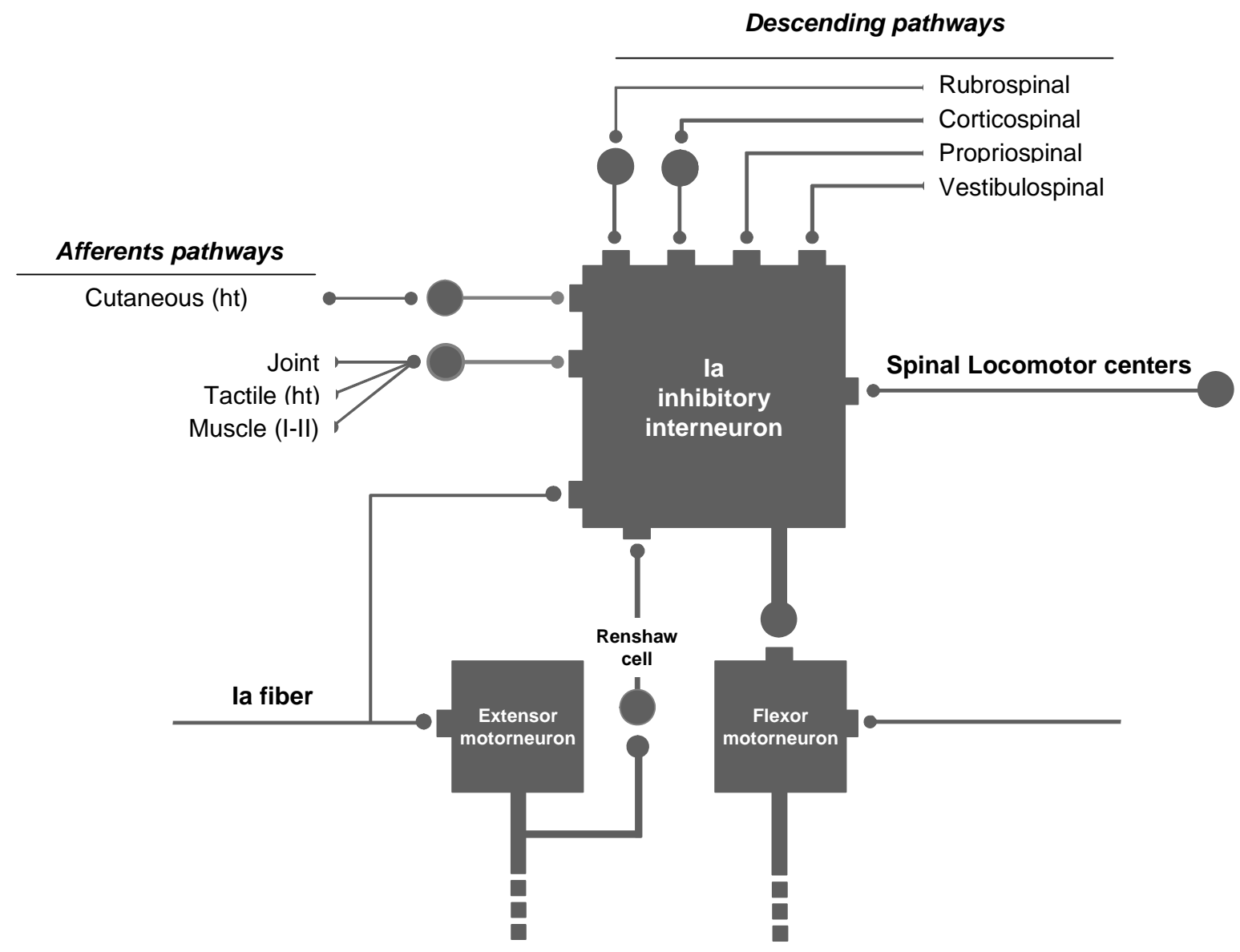

Fig. (1). The crucial role of spinal interneurons in the control of motorneuronal activity.

the nervous system. The interneuron became subject of debate.

\section{PATHOLOGIC ACTIVATION OF THE MOTOR UNIT}

Motorneurons and the associated muscle fibers can develop abnormal activities due to an alteration of descending motor pathways. After transection of cat's brain stem above the level of vestibular nuclei Sherrington observed an enhancement of stretch reflexes and a pronounced increase of the tone in the antigravitary extensor muscles [2]. Even if this kind of rigidity is not marked in humans and in fact was described by Sherrington in the cat, a good definition of spasticity is that given by Peter Nathans " A condition in which stretch reflexes that are normally latent become obvious. The tendon reflexes have a lowered threshold to tap, the response of the tapped muscle is increased, and usually muscles besides the tapped one respond; tonic stretch reflexes are affected in the same way" [3].

An enhancement of stretch reflex may occur following a hyperexcitability of the alpha motorneurons and/or an increase of the excitatory input evoked by muscle stretch [4]. In a more general context, a motorneuron becomes hyperexcitable if depolarizing currents across its membrane are more intense than the hyperpolarizing ones. Several factors may generate motorneuron hyperexcitability: a reduced inhibitory input, supersensitivity following denervation, shortened motorneuron dendrites, collateral sprouting originated by dorsal root afferents.

The upper motorneuron syndrome is characterized by a prevalence of excitatory on the inhibitory inputs. This condition can be induced by cerebral palsy, multiple sclerosis, traumatic brain injury, stroke, spinal cord injury or neurodegenerative diseases [4].

A symptom characteristic of this disease is the spasticity, a motor disorder characterized by a velocity-dependent increase in tonic stretch reflexes with exaggerated tendon jerks, resulting from hyperexcitability [4]. The stiffness of muscles is approximately twice the normal and fibers present some structural alterations [5]. In fact, "spastic" sarcomeres are shorter compared with the normal ones.

The plasticity of neural circuitries can be modified during a motor skill training as well as during a prolonged immobilization. These changes can be detected by recording the magnitude of H-reflexes in humans [6-9]. More specifically, part of the increase in the size of the H-reflex could depend on changes in presynaptic inhibition at spinal level just where information running on Ia afferents are modulated by local interneurons $[10,11]$. In fact, following two weeks immobilization of the left foot and ankle joint in humans, it has been observed a decrease of the plantar 
flexion torque; on the other hand the soleus H-reflex magnitude increased significantly. This last phenomenon could be attributed to a decrease of the presynaptic inhibition on Ia afferents operated by higher motor centers (cortex activity during a motor task or a motor learning) on GABAergic spinal interneurons [12]. From a functional and clinical point of view these results are relevant when considering the effects of a reduced motor activity. An impairment of GABAergic interneurons in pathological states inducing spasticity is consistent with the short-term benefits of baclofen, a $\mathrm{GABA}_{\mathrm{B}}$ receptor agonist, in reducing some symptoms of spasticity, as increased muscle tone and hyperreflexia. On the other hand the clinical observation that in the long term the effect of this drug is to make the whole muscle weaker and fatigable [13], indicates that a simple restoring of inhibitory circuits is inadequate to assure an efficient rehabilitation.

Anyway, during recovery following an incomplete spinal cord injury the muscle strength and amplitudes of motor evoked potentials increase significantly in correlation with significant improvement of the maximal movement velocity. The latencies of the responses continue to be delayed and the background EMG is stable, but the ability to accurately time movements is preserved [14]. Even if these clinical data give no definitive indication of the mechanisms inducing recovery, they suggest a that an improved synchronization of descending volleys could be combined to a higher responsiveness of motorneurons to supraspinal input

In vitro studies on the electrophysiology of motorneurons have been performed in spinal cord of "spastic" mice. Biscoe and Duchen [15] compared the responses of motorneurons in the normal and spastic cord, respectively, by stimulating segmental dorsal root. Motorneurons of the mutant spastic cord exhibited a complex monosynaptic response with a component of asynchronous compound action potentials. In some cases the depolarizing wave showed oscillations that attenuated after $500 \mathrm{msec}$. Intracellular recordings performed to measure membrane parameters (resting potential, inputresistance and voltage-dependent conductance) of motorneurons revealed no difference between normal and spastic mice. Segmental dorsal root stimulation evoked overexpression of orthodromic responses in motorneurons of mutant mice. In fact, stimulation of L4 dorsal root induced a depolarizing synaptic response in L4 spastic motorneurons often able to generate multiple action potentials even before threshold was reached by the monosynaptic response.

Control experiments carried out to verify the involved currents in the abnormal motor output of the spastic mutant revealed a reduction of a glycine-mediated synaptic conductance (chloride current). A question arises to understand whether the mutation interacts with the process encoding glycine receptor proteins or whether the problem is due to a reduced amount of receptors.

A consequence of the spasticity syndrome observed in animal preparations and in humans consists of involuntary muscle contractions (spasms) produced by $\mathrm{Na}^{+}$and $\mathrm{Ca}^{2+}$ inward currents $[16,17]$. Changes, induced in post-synaptic potentials (PSPs) after brief cutaneo-muscular stimulation applied to the second toe or to the medial arch, were studied by Norton et al. [17] in humans with spinal cord injury and compared to data recorded in intact subjects. In non-injured humans single pulse or short train $(<20 \mathrm{msec})$ are able to evoke a long lasting (up to $300 \mathrm{msec}$ ) increase of the firing rate with an interposed period of decreased firing. Similarly, experiments performed on motorneurons of intact rats showed a prolonged excitatory post-synaptic potential with a fast intervening inhibitory post-synaptic potential. The same stimulation applied to spinal cord-injured subjects produced long-lasting (up to $1200 \mathrm{msec}$ ) excitatory potentials with no inhibition interposed. In motorneurons such a duration of depolarization is able to generate a persistent inward current, that is a consistent component of motoneuronal activation in involuntary muscle spasm.

Bennett et al. [18] reported that motorneurons of rats after chronic spinal cord injury exhibit a sustained discharge powered by an "intrinsic plateau". From a functional point of view this plateau and the increase of the discharge contribute to the hypertonus and to the exaggeration of the reflexes associated with spasticity. Thus, these plateaus play an important role since they appear in awake chronic spinal preparates and participate in the generation of muscle spasms.

In spinal astrocytes of rats, ischemic paraplegia induced overexpression of glutamate receptors 1 , a result that could be related to behavioral signs of spasticity and rigidity [19]. In fact, experimental data suggest an involvement of spinal AMPA receptors in spasticity/rigidity. As the increase of these receptors in astrocytes could facilitate the abnormal activity of alpha motorneurons astrocytes can represent one of the new targets for the treatment of the spasticity/rigidity. In this regard, a problem arises about the distinction between neuronal and astrocytic AMPA receptors.

In subjects with hemiparetic stroke the rise time of EPSPs evoked in motorneurons by tendon taps is shorter in the spastic than in the intact side even if the amplitudes are similar [20]. These shortened rise times account for the hyperexcitable reflex responses at short latency.

\section{CONCLUDING REMARKS}

Motor neurons generate sequences of action potentials whose intensity and duration is the integration of inward and outward currents crossing their membranes. The firing of these motoneurons will activate the motor units and, on a macroscopic scale, will induce the muscle contraction.

Unfortunately, motoneurons are not able to distinguish between events that determine their spiking activity. So, muscle iperactivity (spasticity/rigidity) is not a pathologic event for neurons but for the outcoming results!

\section{REFERENCES}

[1] Allen GI, Tsukahara N. Cerebrocerebellar communication systems Physiol Rev 1974; 54(4): 957-1006.

[2] Sherrington C. The integrative action of the nervous system. New Haven, CT, USA: Yale University Press 1906.

[3] Nathan P. Some comments on spasticity and Rigidity. New Developments in Elecromyography and Clinical Neurophysiology $1973 ; 13-4$.

[4] Mayer NH. Clinicophysiologic concepts of spasticity and motor dysfunction in adults with anupper motorneuron lesion. Muscle Nerve 1997; (Suppl) 6: 1-13.

[5] Foran JR, Steinman S, Barash I, et al. Structural and mechanical alterations in spastic skeletal muscle. Dev Med Child Neurol 2005; 47(10): 713-7. 
[6] Gruber M, Taube W, Gollhofer A, Beck S, Amtage F, Schubert M. Training-specific adaptations of $\mathrm{H}$ - and stretch reflexes in human soleus muscle. J Mot Behav 2007; 39(1): 68-78.

[7] Meunier S, Kwon J, Russmann H, Ravindran S, Mazzocchio R, Cohen L. Spinal use-dependent plasticity of synaptic transmission in humans after a single cycling session. J Physiol 2007; 579(Pt 2): 375-88.

[8] Perez MA, Lungholt BK, Nielsen JB. Presynaptic control of group Ia afferents in relation to acquisition of a visuo-motor skill in healthy humans. J Physiol 2005; 568(Pt 1): 343-54.

[9] Perez MA, Lundbye-Jensen J, Nielsen JB. Task-specific depression of the soleus H-reflex after cocontraction training of antagonistic ankle muscles. J Neurophysiol 2007; 98(6): 3677-87.

[10] Hultborn H, Meunier S, Morin C, Pierrot-Deseilligny E, et al. Assessing changes in presynaptic inhibition of I a fibres: a study in man and the cat. J Physiol 1987; 389: 729-56.

[11] Hultborn H, Meunier S, Pierrot-Deseilligny E, Shindo M. Changes in presynaptic inhibition of Ia fibres at the onset of voluntary contraction in man. J Physiol 1987; 389: 757-72.

[12] Lundbye-Jensen J, Nielsen JB. Immobilization induces changes in presynaptic control of group Ia afferents in healthy humans. J Physiol 2008; 586(Pt 17): 4121-35.

[13] Thomas CK, Häger-Ross CK, Klein CS. Effects of baclofen on motor units paralysed by chronic cervical spinal cord injury. Brain 2010; 133(1): 117-25.
[14] Wirth B, Van Hedel HJ, Curt A. Changes in corticospinal function and ankle motor control during recovery from incomplete spinal cord injury. Neurotrauma 2008; 25(5): 467-78.

[15] Biscoe TJ, MR Duchen. Synaptic physiology of spinal motorneurones of normal and spastic mice: an in vitro study. J Physiol 1986; 379: 275-92.

[16] Maynard FM, Karunas RS, Waring WP, 3rd. Epidemiology of spasticity following traumatic spinal cord injury. Arch Phys Med Rehabil 1990; 71(8): 566-9.

[17] Norton JA, Bennett DJ, Knash ME, Murray KC, Gorassini MA. Changes in sensory-evoked synaptic activation of motorneurons after spinal cord injury in man. Brain 2008; 131(Pt 6): 1478-91.

[18] Bennett DJ, Li Y, Harvey PJ, Gorassini M. Evidence for plateau potentials in tail motorneurons of awake chronic spinal rats with spasticity. J Neurophysiol 2001; 86(4): 1972-82.

[19] Hefferan MP, Kucharova K, Kinjo K, et al. Spinal astrocyte glutamate receptor 1 overexpression after ischemic insult facilitates behavioral signs of spasticity and rigidity. J Neurosci 2007; 27(42): 11179-91.

[20] Suresh NL, Ellis MD, Moore J, Heckman H, Rymer WZ. Excitatory synaptic potentials in spastic human motorneurons have a short rise-time. Muscle Nerve 2005; 32(1): 99-103.

Received: September 22, 2009

Revised: April 23, 2010

Accepted: April 23, 2010

(C) Volsi and Licata; Licensee Bentham Open

This is an open access article licensed under the terms of the Creative Commons Attribution Non-Commercial License (http: //creativecommons.org/licenses/by$\mathrm{nc} / 3.0 /$ ) which permits unrestricted, non-commercial use, distribution and reproduction in any medium, provided the work is properly cited. 